Z Epileptol 2015 $\cdot 28: 245$

DOI 10.1007/s10309-015-0019-0

Online publiziert: 10. Juli 2015

(c) Springer-Verlag Berlin Heidelberg 2015

CrossMark

Gerhard Luef

Abteilung für Neurologie, Medizinische Universität Innsbruck, Innsbruck, Österreich

\title{
Epilepsie und Endokrinologie
}

\section{Ein Wechselspiel von epileptischen Anfällen und epileptischer Erregbarkeit, Antikonvul- siva Therapie und Hormonsekretion}

sprüchlich gesehen, daher werden von Prof. Herzog die aktuellsten pathophysiologischen Erkenntnisse und darüber hinaus die Möglichkeit der Progesteron Therapie nach aktuellsten wissenschaftlichem Stand erläutert. Es sollten hier jedoch nicht nur Neurologen zu Wort kommen, daher wurden mit Prof. Dr. Ludwig Wildt und Dr. Benjamin Rösing zwei sehr erfahrene gynäkologische Endokrinologen eingeladen aus ihrer Sicht zum Thema Frauen und Epilepsie zu schreiben. Dieses Themenheft fokussiert jedoch auch die Komplikationen der Behandlung von Epilepsien mit antikonvulsiver Therapie. Die antikonvulsive Medikation kann einige bedeutsame Nebenwirkungen in Bezug auf sexuelle und reproduktive Funktionen als auch auf die Knochendichte haben. Da diese Komplikationen schon möglichst früh erkannt und beleuchtet werden müssen, freut es mich, dass mein langjähriger wissenschaftlicher Partner Priv. Doz. Dr. Markus Rauchenzauner den Teil der Endokrinologie in der Pädiatrie übernommen hat. Da zu guter Letzt all den klinischen Studien experimentelle Daten vorangehen, hat Erik Taubøll mit seinen Mitarbeitern die experimentellen Modelle zu hormonellen Veränderungen bei Epilepsien für dieses Themenheft der Zeitschrift für Epileptologie zusammengefasst und wurden von mir an den Beginn gesetzt.

Ich hoffe, dass die vorliegenden Übersichten Ihr Interesse wecken und in Ihrer Therapieplanung Gebrauch finden werden.

Ihr

Gerhard Luef

\section{Korrespondenzadresse}

Ao. Univ. Prof. Dr. G. Luef

Abteilung für Neurologie, Medizinische Universität Innsbruck

Anichstrasse 35, 6020 Innsbruck, Österreich

gerhard.luef@i-med.ac.at

Interessenkonflikt. G. Luef gibt an, dass kein Interessenkonflikt besteht. 